\title{
Changes in inertia and effect on turning effort across different wheelchair configurations
}

\author{
Jayme J. Caspall, MS; ${ }^{1}$ Erin Seligsohn; ${ }^{1-2}$ Phuc V. Dao, MS; ${ }^{1-2}$ Stephen Sprigle, PhD, PT ${ }^{1-3^{*}}$ \\ ${ }^{1}$ Rehabilitation Engineering and Applied Research Laboratory, ${ }^{2}$ Department of Mechanical Engineering, and ${ }^{3}$ School \\ of Applied Physiology, Georgia Institute of Technology, Atlanta, GA
}

\begin{abstract}
When executing turning maneuvers, manual wheelchair users must overcome the rotational inertia of the wheelchair system. Differences in wheelchair rotational inertia can result in increases in torque required to maneuver, resulting in greater propulsion effort and stress on the shoulder joints. The inertias of various configurations of an ultralightweight wheelchair were measured using a rotational inertia-measuring device. Adjustments in axle position, changes in wheel and tire type, and the addition of several accessories had various effects on rotational inertias. The configuration with the highest rotational inertia (solid tires, mag wheels with rearward axle) exceeded the configuration with the lowest (pneumatic tires, spoke wheels with forward axle) by $28 \%$. The greater inertia requires increased torque to accelerate the wheelchair during turning. At a representative maximum acceleration, the reactive torque spanned the range of 11.7 to $15.0 \mathrm{~N}-\mathrm{m}$ across the wheelchair configurations. At higher accelerations, these torques exceeded that required to overcome caster scrub during turning. These results indicate that a wheelchair's rotational inertia can significantly influence the torque required during turning and that this influence will affect active users who turn at higher speeds. Categorizing wheelchairs using both mass and rotational inertia would better represent differences in effort during wheelchair maneuvers.
\end{abstract}

Key words: bout, caster scrub, manual wheelchair, mobility, propulsion effort, rolling resistance, rotational inertia, turning effort, wheelchair, yaw axis control.

\section{INTRODUCTION}

The typical wheelchair user spends a substantial amount of time performing short maneuvers compared with long, straight maneuvers. This may be an intuitive statement, but it is also supported by the evidence from monitoring wheelchair usage in everyday life. Using the term "bout" to characterize periods of movement between rests, Sonenblum et al. found that the median manual wheelchair user performed about 90 bouts of movement per day, with the median bout lasting $21 \mathrm{~s}$ and traveling $8.6 \mathrm{~m} \mathrm{[1].} \mathrm{Using} \mathrm{these} \mathrm{results} \mathrm{and} \mathrm{a} \mathrm{general}$ understanding of indoor and outdoor environments, one can deduce that maneuvering a wheelchair throughout the day involves frequent changes in direction. As a result, it is important to consider the effort required to turn a wheelchair when assessing the propulsion demand during the execution of common mobility tasks. This article describes a method to measure wheelchair inertia $\left(I_{W C}\right)$ as a means to estimate changes in effort required to turn different configurations of a manual wheelchair.

\footnotetext{
Abbreviations: $\mathrm{FFT}=$ Fast Fourier Transform, $I_{W C}=$ wheelchair inertia, Mobility RERC $=$ Rehabilitation Engineering Research Center on Wheeled Mobility.

*Address all correspondence to Stephen Sprigle, PhD, PT; Rehabilitation Engineering and Applied Research Laboratory, Georgia Institute of Technology, Atlanta, GA 30032-0156; 404385-4302; fax: 404-894-9320. Email: sprigle@gatech.edu http://dx.doi.org/10.1682/JRRD.2012.12.0219
} 
Inertia is characteristic to all objects with mass and reflects the relative tendency of an object to resist any change in its motion. With respect to wheelchairs, the force required to accelerate the wheelchair in a straight trajectory depends on its overall mass (i.e., rectilinear inertia), the rolling resistance of the tires, and other frictional influences. Similarly, the force required to turn a wheelchair depends on rotational inertia and frictional forces. The total turning effort, $T_{\text {applied }}$, applied by the wheelchair operator is the sum of two dominating factors affecting wheelchairs in turning, namely, resistance torque, $T_{\text {resistance }}$, and reactance torque, $T_{\text {reactance }}($ Equation (1)):

$$
T_{\text {applied }}=T_{\text {resistance }}+T_{\text {reactance }} .
$$

The resistance torque results from nonconservative, or frictional, factors such as rolling resistance $\left(T_{R_{\text {roll }}}\right)$, bearing resistance $\left(T_{R_{\text {bearing }}}\right)$, drive wheel scrub $\left(T_{\text {scrub }_{D W}}\right)$, and caster scrub $\left(T_{\text {scrub }_{C}}\right)$. Additional less significant terms, such as drag, frame joint losses, and cushion viscosity, may also be included ( $\left.T_{\text {other }}\right)$ (Equation (2)):

$T_{\text {resistance }}=T_{R_{\text {roll }}}+T_{R_{\text {bearing }}}+T_{\text {scrub }_{D W}}+T_{\text {scrub }_{C}}+T_{\text {other }}$.

As indicated in Equation (3), the reactance torque results from conservative (spring-like) forces ( $\left.T_{\text {conservative }}\right)$ and inertia terms, namely, the rigid body yaw rotational inertia of the wheelchair assembly $\left(T_{I_{W C}}\right)$, drive wheel spin rotational inertia $\left(T_{I_{D W}}\right)$, caster yoke assembly rigid body yaw rotational inertia $\left(T_{I_{\text {yoke }}}\right)$, caster wheel spin rotational inertia $\left(T_{I_{\text {caster }}}\right)$, and the rigid body yaw rotational inertia of the wheelchair occupant $\left(T_{I_{\text {occ }}}\right)$. The yaw axis is the vertical axis normal to the nominally horizontal rolling plane, and the wheel spin axes are aligned with the wheel axles:

$T_{\text {reactance }}=T_{\text {conservative }}+T_{I_{W C}}+T_{I_{D W}}+T_{I_{\text {yoke }}}+T_{I_{\text {caster }}}+T_{I_{\text {occ }}}$.

Both the resistance torque and the reactance torque are greatly influenced by the occupant. The ultralightweight class of wheelchairs is limited to $30 \mathrm{lb}(13.6 \mathrm{~kg})$. The occupant may be up to $250 \mathrm{lb}(113 \mathrm{~kg})$. In typical circumstances, therefore, the inertia of the occupant is much greater than the $I_{W C}$, i.e., $I_{o c c}>I_{W C}$. It is the rectilinear inertia, i.e., mass, of the occupant that drives the normal forces on bearing surfaces that lead to frictional loads. Likewise, it is the rigid body yaw rotational inertia of the occupant that dominates the reactance torque required for turning.
In spite of the fact that the inertia of the occupant dominates the reactance torque, changes in the rigid body yaw rotational inertia of the wheelchair assembly $\left(I_{W C}\right)$ are not negligible. This fact motivates wheelchair manufacturers to provide ever lighter frames in a competition of optimization. This fact also motivated this article, with the objective to characterize the differences in changes in the rigid body yaw rotational inertia of the wheelchair assembly $\left(I_{W C}\right)$ resulting from variations in the wheelchair configuration.

The reactance torque includes flexibility of the frame (in the term $T_{\text {conservative), }}$, but because of the rigid character of the frame construction most commonly encountered in ultralightweight wheelchairs, the dominant reactance is due to the inertial properties of the wheelchair components. Of these, the rigid body yaw rotational inertia of the occupant and the rigid body yaw rotational inertia of the wheelchair assembly are most significant, i.e., Equation (4):

$$
T_{\text {reactance }} \cong T_{I_{\text {occ }}}+T_{I_{W C}}
$$

Given a yaw angular acceleration $(\alpha)$, we have Equation (5):

$$
T_{I_{o c c}}+T_{I_{W C}}=\left(I_{o c c}+I_{W C}\right) \alpha .
$$

A higher yaw moment of inertia requires a greater effort to initiate (or stop) turning motion. Both resistance torque and reactance torque act in opposition to the userapplied torque. Reduction of one or both of these factors leads to a reduction in the propulsion effort required for turning. Only changes in $I_{W C}$ because of adjustable configuration changes were considered in this article.

Several studies offer insight into the magnitudes of different frictional and inertia factors. For example, Hofstad and Patterson reported that the effect of bearing resistance is insignificant in wheelchair propulsion effort when compared with inertia or rolling resistance [2]. Kauzlarich and Thacker determined that, given the same load and same wheel material, rolling resistance is inversely proportional to wheel radius [3]. In part because of this relationship, ultralightweight wheelchairs are set up with a majority of load over the drive wheels, typically exceeding 70 percent [4]. In addition to rolling, casters have an additional degree of freedom of rotation about the caster stem. As a result, some of the user propulsion effort goes into overcoming caster scrub as the casters swivel during turning maneuvers. 
Given that caster resistance is specifically pertinent in turning maneuvers, inertial reactance should be compared with an estimate of the caster scrub turning resistance, which is the resistance experienced when casters swivel on the ground. To investigate the relative contribution of caster scrub to the turning resistance, we derived a comparative estimate from the quantitative work of Frank and Abel [5]. They studied the caster scrub effect on various typical hospital and nursing home floor surfaces. They used a custom testing apparatus to determine the torque required to pivot a wheel about a vertical axis passing through the point of contact of the tire and the floor. They tested three different flooring materials and a variety of caster wheels having diameters ranging from 100 to $200 \mathrm{~mm}$ and effectively measured the result of dynamic friction by measuring the torque under a constant rotation rate. By changing the normal load through the point of contact between the tire and the floor, they obtained curves for the load-dependent turning resistance for a caster over a load range of 50 to $300 \mathrm{~N}$. The turning resistances spanned the range from about 0.3 to about $2.8 \mathrm{~N}-\mathrm{m}[5]$.

A few studies are aimed at relating the inertial reactance to user propulsion. Brubaker looked at how mobility is affected by changes in the axle position [6]. He found that moving the rear wheel axle shifts the weight distribution on the rear and caster wheels, which has a significant effect on what he called "yaw axis control." This yaw axis control is defined as "the forces required to maneuver the wheelchair." The turning resistance curves from Frank and Abel indicate a doubling of the resistance from a caster load of $100 \mathrm{~N}$ to a caster load of $160 \mathrm{~N}$ [5]. From this, it is clear that an increase in normal load on the caster yoke stem has a significant effect on yaw axis control. MacPhee et al. looked at how body positioning, specifically the knee-flexion angle, affects turning effort [7]. It turned out that with the knees fully flexed, the turning velocity was increased by 40 percent and user-perceived effort was 66 percent easier in turning as compared with knees in full extension. The way mass is distributed in the system, therefore, has a great effect on turning effort.

The magnitude of the turning effort ultimately affects the long-term health of wheelchair users. According to Mercer et al., higher forces exerted by the shoulder increase the risk of shoulder pathology, which means increased risk of shoulder injuries such as impingement syndrome [8]. Any factor that serves to reduce the turning effort will reduce the load on the shoulder and result in lower exposure levels for each executed maneuver, thereby reducing the risk of shoulder injury.

The rotational inertia of manual wheelchairs is important because even a slight mass redistribution within the system can cause an affective change in required propulsion effort. To determine the turning effort of wheelchairs, it is vital to have data on both resistance torque and rotational inertia of the wheelchair. The inertial properties of a system depend on the mass, and for turning maneuvers, on the mass distribution, or $I_{W C}$. For example, changing the axle position can change $I_{W C}$ without a change in mass. This article measures the changes in inertial reactance or in $I_{W C}$ resulting from configuration changes in adjustable manual wheelchairs and relates inertial changes to differences in the torque required to overcome caster scrub and to accelerate wheelchairs during turning.

\section{METHODS}

\section{Inertial Measurements}

To characterize the effect of wheelchair configurations on $I_{W C}$, a TiLite Aero $\mathrm{Z}$ manual wheelchair (Kennewick, Washington) was used as the test base. This wheelchair is in the ultralightweight class and offers several adjustable features and optional configurations to allow the chair to be individualized to suit the user's needs. We decided to focus on three independent variables reflecting configurations relevant to changes in rotational inertia: (1) fore-aft rear axle position, (2) tires and wheels, and (3) the addition of three accessories: armrests, antitip bars, and clothing shields. Definition and illustration of the different configurations are contained in the Table and Figure 1, respectively. This study focused on the effect of configuration changes to the wheelchair itself; therefore, the mass of the wheelchair user was not included in measuring inertia. Also, the moment of inertia of the wheels about their axles is neglected in order to narrow the study's focus on yaw inertia. The inclusion of both variables is suggested for future studies.

Practically speaking, one cannot independently calculate the inertia of every frame, component, and configuration. For this reason, $I_{W C}$ was measured experimentally using the inertia measurement device called the iMachine [9]. We developed the iMachine to measure $I_{W C}$ for irregularly-shaped rigid bodies, specifically, an occupied 
Table.

Masses and moments of wheelchair inertia $I_{w c}$. Wheelchair configurations listed in order of increasing moment of inertia.

\begin{tabular}{|c|c|c|c|c|c|c|}
\hline Configuration & Wheels & Accessories & $\begin{array}{c}\text { Axle } \\
\text { Position }\end{array}$ & $\begin{array}{c}\text { Mass } \\
\text { (kg) }\end{array}$ & $\begin{array}{c}\text { Mean } I_{W C} \\
\left(\mathbf{k g}-\mathbf{m}^{2}\right)\end{array}$ & $\begin{array}{l}\text { Coefficient of } \\
\text { Variance (\%) }\end{array}$ \\
\hline 1 & Pneumatic Spoke & No & Aft & 11.36 & 1.1672 & 0.57 \\
\hline 9 & Solid Mag & No & Aft & 12.40 & 1.2626 & 0.38 \\
\hline 4 & Pneumatic Spoke & Yes & Fore & 13.06 & 1.2734 & 0.36 \\
\hline 3 & Pneumatic Spoke & Yes & Aft & 13.06 & 1.2946 & 0.63 \\
\hline 12 & Solid Mag & Yes & Fore & 14.10 & 1.3629 & 0.42 \\
\hline 11 & Solid Mag & Yes & Aft & 14.10 & 1.3880 & 0.37 \\
\hline 8 & Primo Solid Mag & Yes & Fore & 14.82 & 1.4315 & 0.87 \\
\hline 7 & Primo Solid Mag & Yes & Aft & 14.82 & 1.4663 & 0.64 \\
\hline
\end{tabular}

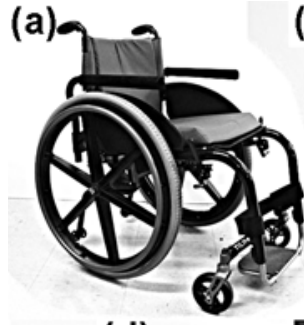

(b)
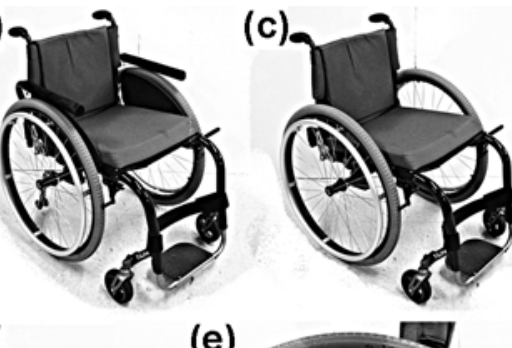

(d)

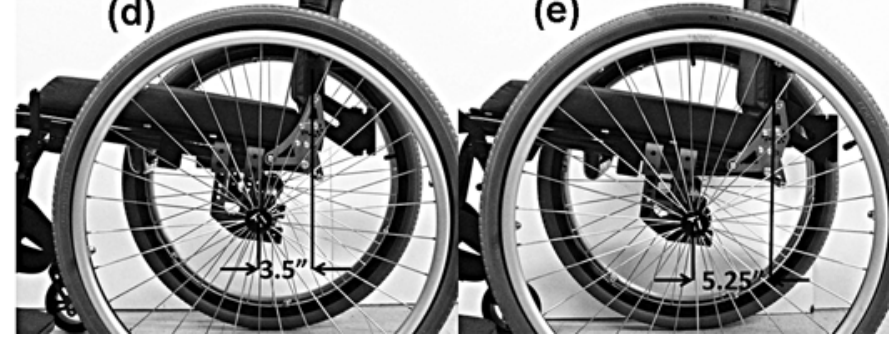

Figure 1.

Wheelchair configurations: (a) solid tires and mag wheels with accessories, (b) pneumatic tires and spoke wheels with accessories, (c) pneumatic tires and spoke wheels with no accessories, (d) aft axle position, and (e) fore axle position.

wheelchair rotating about the yaw axis. The device consists of a turntable mounted to a base with a single axle. Load cells mounted on the turntable measure the mass and center of mass of the wheelchair. An encoder measures rotation of the turntable, whose oscillations are damped by a spring of known stiffness. For the purposes of this study, the need to measure unoccupied wheelchairs necessitated a modification of the iMachine. A lighter platform with a lower $I_{W C}\left(0.267 \mathrm{~kg}-\mathrm{m}^{2}\right.$, compared to $\left.3.452 \mathrm{~kg}-\mathrm{m}^{2}\right)$ was constructed. This platform also eliminated an adjustable platform so the inertia of the turntable was constant. Testing using objects with analytically determined inertias found error within 0.82 percent. Other than this modification, the iMachine used in this study was identical to the apparatus reported in Eicholtz et al., and specific information about the design and technique can be found there [9].

Briefly, the wheelchair to be measured was positioned onto the iMachine and load cells measured the mass and center of mass. The distance between the wheelchair's center of mass and the axis of rotation of the iMachine was used to apply the parallel axis theorem when calculating the yaw moment of inertia about the wheelchair's center of mass. The sprung turntable was given an initial displacement and released. A time series of angle positions was sampled continuously at a rate of $45 \mathrm{~Hz}$ during the damped oscillator response. The time series was transformed to the frequency domain by applying a Fast Fourier Transform (FFT), and the peak of the FFT was found by fitting a parabola to the peak of the spectral response. The location of the peak value of the parabola was used as the resonance frequency value $\left(\omega_{n}\right)$ in the determination of the inertia using Equation (6):

$$
I_{W C}=\frac{k_{\theta}^{2}}{\omega_{n}^{2}}-I_{0}
$$

where $k$ is the known spring constant and $I_{0}$ is the inertia of the unloaded iMachine turntable. This measurement was repeated five times for each configuration. 
A $t$-test was used to assess inertia differences between forward and rear axle positions. To better understand the clinical significance of inertial differences across all configurations, $I_{W C}$ was estimated. $T_{I_{W C}}$ is the product of this inertia measurement and the angular acceleration. In order to understand the significance of the total turning reactance, a maximum value for the wheelchair acceleration during turning was needed.

A test was conducted to estimate the maximum wheelchair turning acceleration that would be used to calculate $T_{I_{W C}}$. A highly active, full-time wheelchair user performed a zero-radius turn using the TiLite Aero $\mathrm{Z}$ chair. Each turn was performed with three to four pushes. A LEGO NXT programmable brick (Billund, Denmark) with a connected gyro sensor was used to measure rotation rate. The NXT gyro sensor measured single-axis rotation up to a rate of $\pm 360 \%$ s. Both the brick and gyro sensor were mounted to the TiLite Aero $\mathrm{Z}$ chair with the gyro sensor rigidly attached to the chair to ensure accurate measurements (Figure 2).

The NXT brick was programmed to sample and record the gyro at a rate of 25 samples per second. The recorded data were transferred to a computer via USB connection. The NXT software v2.1 was used to visualize the data. The derivatives of the recorded rotation rate time series were calculated using a first-order finite difference approximation in MATLAB (MathWorks; Natick, Massachusetts). The peaks of the resulting angular acceleration were determined, and the mean of five

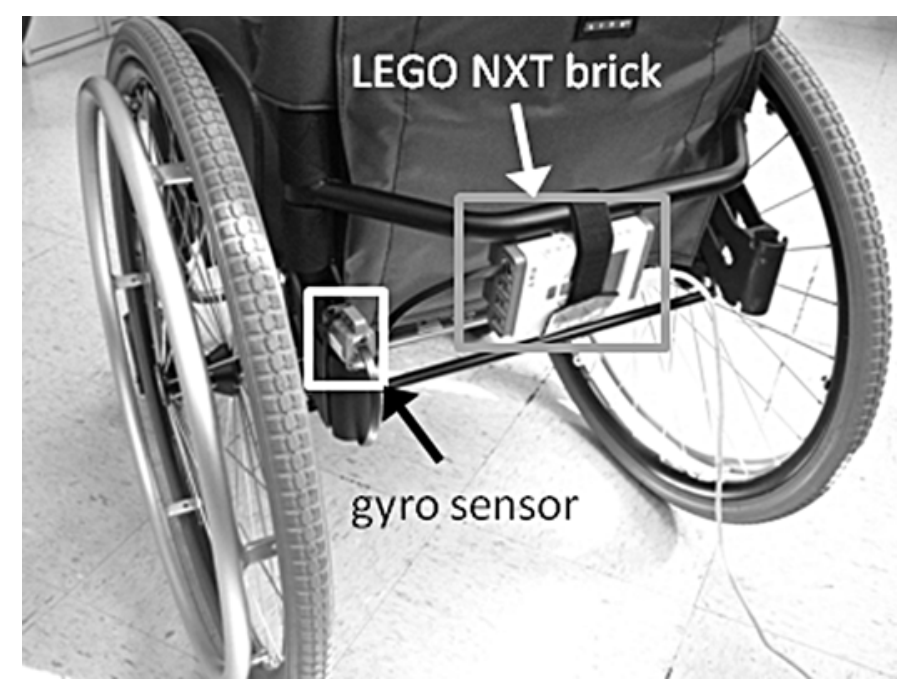

Figure 2.

LEGO NXT setup. separate measurements was calculated to serve as the representative maximum turning acceleration. This measured maximum angular acceleration was intended to serve as a representative maximum turning acceleration likely to be experienced by a manual wheelchair user during quotidian use.

\section{Turning Resistance from Caster Scrub}

Calculation of turning resistance from caster scrub was needed to gauge the relative effect of changes in inertial reactance of the different wheelchair configurations. Using the results from Frank and Abel [5], the caster scrub resistance was estimated for a generalized wheelchair undergoing a zero-radius turn (drive wheels driven equally in opposite directions) (Figure 3) for a chair in this configuration. For this zero-radius turn, the yaw axis was defined as being at the midpoint between the two drive wheels, as shown. While not all wheelchair turns are zero radius, modeling a zero-radius turn affords a straightforward calculation of caster swivel that leads to caster scrub. During wheelchair maneuvers, the trajectory of the chair involves both translational and rotational

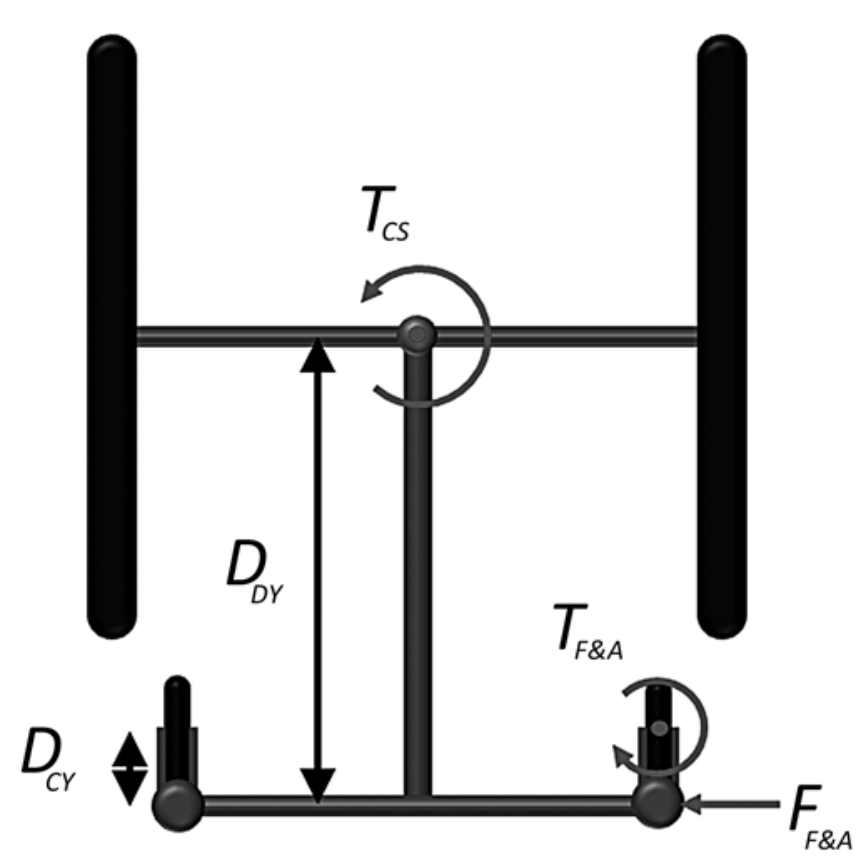

Figure 3.

Schematic of wheelchair parameters used in estimating caster scrub torque. $D_{C Y}=$ caster trail, $D_{D Y}=$ length of moment arm, $F_{F \& A}=$ force required to swivel caster, $T_{C S}=$ resistance torque from caster scrub, $T_{F \& A}=$ torque obtained from Frank and Abel study [5]. 
movements. The casters will swivel to become tangent to the arc of the turning wheelchair, and that resulting caster swivel results in the caster scrub being calculated here.

The force required to swivel a caster is $F_{F \& A}=T_{F \& A} /$ $D_{C Y}$, where $T_{F \& A}$ is the torque obtained from the Frank and Abel study [5] and $D_{C Y}$ is the caster trail. This force acts through a moment arm of length $\left(D_{D Y}\right)$ resisting the turning torque applied about the center of the line between the two drive wheels. The resistance torque from caster scrub, $T_{C S}$, that must be overcome by the operator is then computed using Equation (7), where the factor of two accounts for two casters in the wheelchair.

$$
T_{C S}=2 \times T_{F \& A}\left(\frac{D_{C Y}}{D_{D Y}}\right)
$$

This resistance torque occurs whenever the casters swivel and is referred to as caster scrub. Caster scrub is present until the casters align tangent to the radius of the turn. The peak resistance torque from caster scrub for a zero-radius turn may be approximated by assuming wheelchair configuration and load conditions. Calculations assumed a caster trail of $4.0 \mathrm{~cm}$, a normal load of $100 \mathrm{~N}$ on each caster (using a 20/80 caster-to-drive wheel weight distribution for a $90 \mathrm{~kg}$ user and a $12 \mathrm{~kg}$ wheelchair), and a caster yoke stem to drive wheel contact distance, $D_{D Y}$, of $35 \mathrm{~cm}$. Frank and Abel found that caster scrub is constant for a given normal force between the caster and the ground [5]. The calculated turning resistance from caster scrub is approximately $8.75 \mathrm{~N}-\mathrm{m}$. Note that this estimate is based on a user mass that is about 80 percent of the weight capacity of most ultralightweight wheelchairs. Using a lighter or heavier occupant would scale the values accordingly.

\section{RESULTS}

The resonance frequency of the loaded iMachine turntable was measured for the various wheelchair configurations. As indicated in Equation (6), the rotational inertia is inversely proportional to the square of the measured resonance frequency. Figure 4 contains two representative ensembles of five resonance frequencies recorded of wheelchairs configured identically except for two drive wheel axle positions (configurations 1 and 2 in the Table). The results, shown in Figure 4, illustrate the

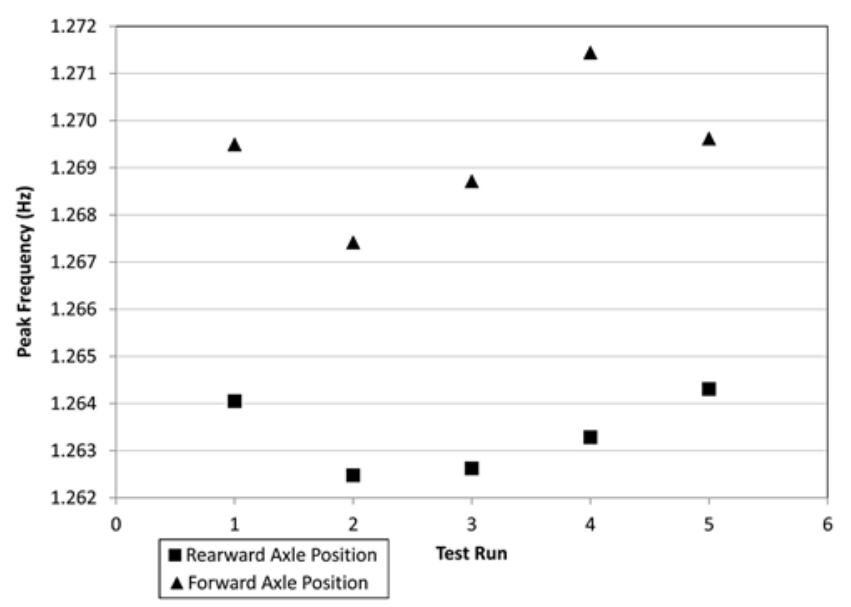

Figure 4.

Measured natural frequencies for two different axle positions.

high precision of the natural frequency measurement and the ability to resolve the difference in frequency caused by the change in axle position. Over five trials, the range of the measured natural frequencies was $0.0041 \mathrm{~Hz}$ for the fore position and $0.0018 \mathrm{~Hz}$ for the aft position. The difference in the means of the two cases was $0.0060 \mathrm{~Hz}$. The measured natural frequency for the rearward axle position was lower than that of the forward axle position, indicating a higher $I_{W C}($ critical $t=2.30, p<0.001)$.

The results for each of the 12 configurations are tabulated in the Table and graphed in Figure 5. Reported inertia and mass values correspond to an average of five separate measurements. The configurations are listed in order of increasing rotational inertia and illustrate a highly linear relationship between the masses and inertias. The small coefficients of variation indicate the high precision of the measurement. In every case, changing the axle position from rearward to forward resulted in a decrease in rotational inertia, averaging 1.9 percent. Removing the accessories resulted in average decreases of 12.1 percent in total mass and of 9.1 percent in $I_{W C}$. Configurations with the lowest and highest inertia (configurations 2 and 7, respectively) differed in mass by 30.5 percent with a 27.7 percent difference in inertia.

To place these results in the context of daily use, a value for the maximum angular acceleration expected by a representative wheelchair user was measured and found to be $\alpha_{\text {rep }}=10.2 \mathrm{rad} / \mathrm{s}^{2}$. Using this result, the reactive torque resulting from $I_{W C}$ may be compared with the resistance torque resulting from caster scrub, a significant component 


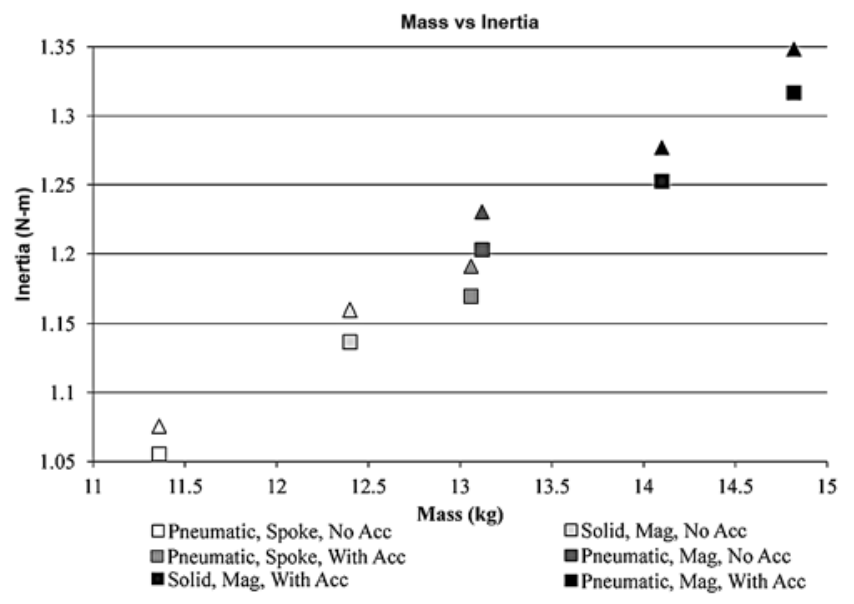

Figure 5.

Inertia vs mass. Acc = accessories. Square and triangle markers indicate forward and rear axle positions, respectively.

of total resistance torque. The reactive torque from the wheelchair rotational inertia is $T_{I W C}=I_{W C} \times \alpha$. A plot of this user-applied reactive torque as a function of turning acceleration is shown in Figure 6. The horizontal line represents the caster scrub resistance torque $=8.75 \mathrm{~N}-\mathrm{m}$ as inferred from the Frank and Abel results presented previously [5]. The two diagonal lines have slopes equal to the measured minimum and maximum inertias of the wheelchair configurations ( 2 and 7 from the Table), and the vertical line corresponds to the representative maximum angular acceleration. So at this acceleration, the wheelchair with lowest inertia configuration (configuration 2) has a reactive torque of $11.7 \mathrm{~N}-\mathrm{m}$, whereas the highest inertia configuration (configuration 7) has a $15.0 \mathrm{~N}-\mathrm{m}$ torque. Note that both exceed the resistance torque of caster scrub, indicating that with rapid turns, the inertia of the wheelchair contributes more turning resistance than caster scrub. The shaded area, therefore, represents the range of angular accelerations expected in everyday wheelchair use, with slow turns near zero on the left side and very rapid turns beyond the right side at some undetermined limit.

\section{DISCUSSION}

The highly linear trend in Figure 5 shows that the measured rotational inertia is strongly correlated with the mass of the wheelchair configuration. Because a single

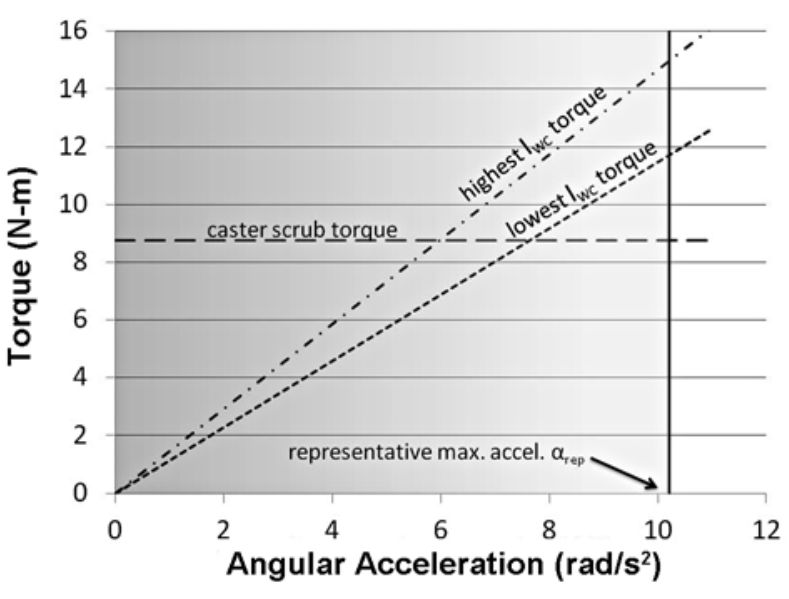

Figure 6.

Comparison of caster scrub torque and reactive torque during turning. $I_{W C}=$ wheelchair inertia, $\max$. accel. = maximum acceleration.

wheelchair frame was used, the configurations shared a high degree of geometrical similarity, making this a rather intuitive result. While the configurations are geometrically similar, the effect of the spatial distribution of the mass is evident between those configurations in which only the axle configuration has changed. The results indicate that a forward axle position reduces the rotational moment of inertia of a wheelchair by about 2 percent. A lower inertia would lead to both a decreased turning effort and lowered pitch stability, a well-known configuration tradeoff. Some users can manage reduced pitch stability, whereas others might face unacceptable instability while climbing inclines and other activities. The effect of the mass distribution can also be seen in the sharp increase in inertia despite only a small increase in total mass (13.06-13.12 kg) between configurations 3 and 4 (pneumatic, spoke, with accessories) and configurations 5 and 6 (Primo solid, mag, no accessories). The heavier mag wheels focus the mass toward the outer edges of the wheelchair (increasing inertia). This effect is similar to that of adding the accessories, which also positions mass toward the outer edges; however, since the wheels are farther from the center of mass, their mass has a more significant effect on inertia.

Figure 6 illustrates the relative turning effort resulting from caster scrub and the wheelchair's rotational inertia. This value resulted from a wheelchair set-up with 20 percent load on the casters and swiveling on a firm tile surface. Greater load on the casters and swiveling on 
different surfaces (i.e., carpeted) will raise this value. Given these caveats, the graph illustrates how a range of angular accelerations during turning will influence turning effort because of rotational inertia. Since the rate of turning is directly related to the turning effort, the manner in which a wheelchair is used will naturally affect forces required to maneuver. For example, a wheelchair user turning slowly would be less likely to notice the reaction difference from the reduced mass and inertia of a wheelchair. Instead, the caster scrub would be much more important. Conversely, for an active user who turns quickly, any reduction in inertia may be noticed. Similarly, differences in a wheelchair's inertia (illustrated by the diverging dotted lines) become increasingly more important when the speed of turning increases. In summary, the change in $I_{W C}$ affected by the change in configurations of an ultralightweight wheelchair is significant for turning accelerations within the range of typical user experience.

Repetitive use shoulder injury has been identified as a chronic problem among full-time manual wheelchair users [8,10-11]. In fact, a wheelchair with lower mass is selected, in part, to reduce the effort required to propel their wheelchairs. Because real-life mobility includes the need to change direction, the wheelchair's yaw moment of inertia also influences effort required to maneuver. Measuring both the mass and yaw moment of inertia would afford a fuller representation of influence on propulsion effort than only using mass, and therefore, would be a better means to characterize differences in technology.

This study measured the changes in inertia across different configuration of a single wheelchair. This approach was selected to isolate the influences of configuration changes rather than changes in the overall wheelchair frame. This focus was also intended as a means to present the methodology and analysis. However, limiting the study to a single ultralightweight wheelchair limits the applicability of the results and indicates the need to study more wheelchair designs and configurations. Similarly, measuring the inertia of unoccupied, nonmoving wheelchairs permitted reporting of inertial changes to the wheelchair alone but comes with the limitation of ignoring the total mass and inertia of an occupied wheelchair system. Wheelchair users not only vary in mass, they also vary in mass distribution because of body type, disability, and posture. All these variables will affect the inertia and will influence frictional factors involved in a moving wheelchair. Future work will measure the summation of reac- tance and resistance forces during wheelchair maneuvers of occupied wheelchairs during free-wheeling maneuvers. That information will combine the inherent influences of mass and inertia with the sources of energy loss due to friction from rolling resistance, caster and drive wheel scrub, and bearings to gain a more complete picture of how turning effort can be reduced when configuring wheelchairs.

\section{CONCLUSIONS}

Currently, wheelchairs are categorized by mass, which represents rectilinear inertia. This study investigated the influence of yaw rotational inertia on turning effort, specifically, resolving the influence across configurations in an ultralightweight wheelchair. The reactance torque related to the yaw moment of inertia of the wheelchair was compared with the resistance torque related to caster scrub. Since this reactance torque is proportional to turning acceleration while the resistance torque is constant, the relative contribution of each varies according to how the wheelchair is maneuvered. During slower turns, the differences in reactance torque across the configurations were found to be comparable to caster scrub. At faster turns, the effects of the inertia changes are more pronounced. For this reason, the effects of wheelchair configuration changes are, generally, more significant for active users and less significant for slow-moving users.

\section{ACKNOWLEDGMENTS}

\section{Author Contributions:}

Study concept and design: J. J. Caspall, S. Sprigle.

Acquisition of data: E. Seligsohn, P. V. Dao.

Analysis and interpretation of data: J. J. Caspall, E. Seligsohn, P. V. Dao, S. Sprigle.

Drafting of manuscript: E. Seligsohn, P. V. Dao.

Critical revision of manuscript for important intellectual content:

J. J. Caspall, S. Sprigle.

Statistical analysis: P. V. Dao.

Obtained funding: S. Sprigle.

Study supervision: J. J. Caspall.

Financial Disclosures: The authors have declared that no competing interests exist.

Funding/Support: This material was based on work supported by the Rehabilitation Engineering Research Center on Wheeled Mobility (Mobility RERC) and the Georgia Institute of Technology. The Mobility RERC is funded by the National Institute on Disability and Rehabilitation Research of the U.S. Department of Education (grant H133E080003). 
Disclaimer: The opinions contained in this article are those of the grantee and do not necessarily reflect those of the U.S. Department of Education.

\section{REFERENCES}

1. Sonenblum SE, Sprigle S, Lopez RA. Manual wheelchair use: Bouts of mobility in everyday life. Rehabil Res Pract. 2012;2012:753165. [PMID:22848837] http://dx.doi.org/10.1155/2012/753165

2. Hofstad M, Patterson PE. Modelling the propulsion characteristics of a standard wheelchair. J Rehabil Res Dev. 1994; 31(2):129-37. [PMID:7965869]

3. Kauzlarich JJ, Thacker JG. Wheelchair tire rolling resistance and fatigue. J Rehabil Res Dev. 1985;22(3):25-41. [PMID:3835263] http://dx.doi.org/10.1682/JRRD.1985.07.0025

4. Asahara S, Yamamoto S. A method for the determination of center of gravity during manual wheelchair propulsion in different axle positions. J Phys Ther Sci. 2007;19(1):57-63. http://dx.doi.org/10.1589/jpts.19.57

5. Frank TG, Abel EW. Measurement of the turning, rolling and obstacle resistance of wheelchair castor wheels. J Biomed Eng. 1989;11(6):462-66. [PMID:2811344] http://dx.doi.org/10.1016/0141-5425(89)90040-X

6. Brubaker CE. Advances in wheelchair technology. IEEE Eng Med Biol Mag. 1988;7(3):21-24. [PMID:18244070] http://dx.doi.org/10.1109/MEMB.1988.999790

7. MacPhee AH, Kirby RL, Bell AC, MacLeod DA. The effect of knee-flexion angle on wheelchair turning. Med Eng Phys. 2001;23(4):275-83. [PMID:11427365] http://dx.doi.org/10.1016/S1350-4533(01)00024-8
8. Mercer JL, Boninger M, Koontz A, Ren D, Dyson-Hudson T, Cooper R. Shoulder joint kinetics and pathology in manual wheelchair users. Clin Biomech (Bristol, Avon). 2006; 21(8):781-89. [PMID:16808992] http://dx.doi.org/10.1016/j.clinbiomech.2006.04.010

9. Eicholtz MR, Caspall JJ, Dao PV, Sprigle S, Ferri A. Test method for empirically determining inertial properties of manual wheelchairs. J Rehabil Res Dev. 2012;49(1):51-62. [PMID:22492337] http://dx.doi.org/10.1682/JRRD.2011.03.0045

10. Janssen TW, van Oers CA, van der Woude LH, Hollander AP. Physical strain in daily life of wheelchair users with spinal cord injuries. Med Sci Sports Exerc. 1994;26:661-70.

11. van der Woude LH, Dallmeijer AJ, Janssen TW, Veeger D. Alternative modes of manual wheelchair ambulation: An overview. Am J Phys Med Rehabil. 2001;80(10):765-77. [PMID:11562560] http://dx.doi.org/10.1097/00002060-200110000-00012

Submitted for publication December 14, 2012. Accepted in revised form May 28, 2013.

This article and any supplementary material should be cited as follows:

Caspall JJ, Seligsohn E, Dao PV, Sprigle S. Changes in inertia and effect on turning effort across different wheelchair configurations. J Rehabil Res Dev. 2013;50(10): 1353-62.

http://dx.doi.org/10.1682/JRRD.2012.12.0219

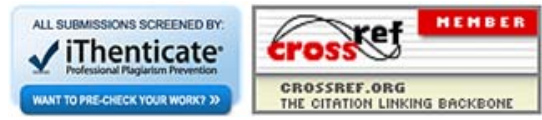


